\title{
El planteamiento teórico multiobjetivo/multicroterio y su aplicación a la resolución de problemas medioambientales y territoriales, mediante los S.I.G. Raster
}

\author{
José Miguel Santos Preciado
}

\begin{abstract}
RESUMEN
La complejidad del medio natural, evidenciada por la intervención de múltiples variables de carácter interactivo, y su respuesta a la acción humana, ha encontrado en la metodología de la "Evaluación Multicriterio" (EMC) un modelo teórico de gran operatividad. El tratamiento masivo de la información geográfica, implícito a un planteamiento de esta naturaleza, ha obligado a la utilización de los Sistemas de Información Geográfica (SIG), como herramienta informática capaz de organizar los datos de forma georreferenciada $y$ evaluar los resultados alcanzados, de manera eficaz, en un tiempo récord. Esta perfecta conjunción entre los SIG y la metodología EMC ha resultado altamente fructífera en el tratamiento $y$ resolución de algunos problemas medioambientales $y$ territoriales. Su aplicación a la valoración de la aptitud medioambiental de un territorio, en el establecimiento de la función

\section{ABSTRACT}

The complexity of the natural environment, shown by the intervention of multiple variables of interactive character and its response to human action, have found in the "Multicriteria Evaluation" methodology (EMC), a theoretical model of great effectivity. The huge dealing with geographical information, which is inherent to an approach of this nature, has forced to the use of the Geographical Information Systems (GIS), as a computer tool able to organize data in a geo-referentiated way and to efficiently evaluate achieved results in record time. This perfect combination of the GIS and the EMC methodology has been highly fruitful in the treatment and solution of some environmental and territorial problems. Its application to the evaluation of a territory's environmental aptitudes, to the establishing of an industrial function, has made it possible for us to offer a general view of the practical
\end{abstract}


industrial, nos ha servido de base para ofrecer un panorama de las posibilidades prácticas de esta interesante metodología. possibilities of this interesting methodology.

\section{INTRODUCCIÓN}

Tradicionalmente, la teoría económica, sobretodo la de carácter microeconómico, se ha apoyado en un planteamiento normativo, que configura a los agentes económicos racionales como a entes que ordenan sus decisiones según la función de un único criterio (en general, el del beneficio). La aparición, durante la década de los años setenta, del denominado paradigma decisional multicriterio, ha transformado este primitivo planteamiento, sustituyéndolo por otro, más complejo, que presupone que las decisiones racionales económicas no se toman en función de un solo objetivo, sino que se busca un equilibrio o compromiso entre un conjunto de objetivos, usualmente en conflicto.

La aplicación del paradigma decisional multicriterio en el campo de la planificación física del territorio ha abierto una interesante vía metodológica al tratamiento de la problemática medioambiental y de la ordenación territorial. La complejidad del medio natural, evidenciada por la intervención de múltiples variables de carácter interactivo, y su respuesta a la acción humana, ha encontrado en la metodología de la "Evaluación Multicriterio" (EMC) un modelo teórico de gran operatividad.

El tratamiento masivo de la información geográfica, implícito a un planteamiento de esta naturaleza, obliga a la utilización de los Sistemas de Información Geográfica (SIG), como herramienta informática capaz de organizar los datos de forma georreferenciada y evaluar los resultados alcanzados, de manera eficaz, en un tiempo récord. Esta perfecta conjunción entre los SIG y la metodología EMC ha resultado altamente fructífera en el tratamiento y resolución de algunos problemas medioambientales y territoriales. Mostrar este camino es el propósito fundamental del trabajo que presentamos.

\section{LA TOMA DE DECISIONES EN EL CONTEXTO TEÓRICO MULTIOBJETIVO Y MULTICRITERIO}

La evaluación multicriterio puede definirse como un conjunto de técnicas orientadas a asistir los procesos de toma de decisión, investigando 
un número de alternativas a la luz de múltiples criterios y objetivos en conflicto (Barredo J.I., 1996).

De acuerdo con este planteamiento, la estimación entre alternativas, concebidas éstas como diferentes hipótesis (para facilitar la exposición, vamos a centrarnos en la referencia a la problemática de la localización geográfica de las actividades humanas en el territorio, siendo las alternativas los potenciales lugares de localización posibles), se realizaría por un proceso de evaluación mono/multiobjetivo '. La existencia de un único objetivo (podría ser la localización en el territorio de la función industrial) facilita la toma decisiones. Sin embargo, lo más normal es que ésta deba realizarse satisfaciendo varios objetivos, complementarios o en conflicto. En el primero de los casos, la combinación de objetivos debe cumplirse al unísono (imagínese la localización de usos del suelo no excluyentes, como la residencia y los servicios, que posibilitaría la existencia de zonas que cumplieran ambos requisitos), mientras en el caso de objetivos conflictivos, éstos se eliminarían entre sí (piénsese como objetivos enfrentados la ubicación de una industria contaminante y la reserva de suelo correspondiente a un parque natural, el área dedicada a un uso sería incompatible con la destinada a otro).

Una vez definidos los objetivos de la evaluación, cada uno de ellos debería ser estimado, mediante la utilización de una serie de criterios o variables. Los criterios deben ser concebidos como los elementos primarios del análisis, cuya combinación permite valorar analíticamente las diversas alternativas para cada uno de los objetivos.

Los criterios pueden ser de dos tipos: factores y restricciones. Un factor es un criterio que permite definirse en favor de una alternativa y en contra de otra, al comparar los resultados de medir, cuantitativamente, el valor alcanzado en cada una de ellas. Siguiendo con el ejemplo anterior, la localización de la industria puede ser evaluada respecto a una serie de factores: proximidad a las vías de comunicación, proximidad a la ciudad, precio del suelo, pendiente suave del espacio, disponibilidad de terrenos superior a una superficie mínima, etc. Por su parte, los criterios restrictivos limitan la posibilidad de considerar alguna alternativa, excluyéndolas de forma definitiva. En el ejemplo que nos ocupa, se podría descartar del territorio apto para la localización industrial, aquel que superara una pendiente mayor del $10 \%$.

Este apartado ha sido elaborado a partir de la obra: "Gis and Decision Making" de J.R. EASTMAN, P.A.K. KYEM, J. TOledANO y W. JiN, recogida en la bibliografía final. 
La toma de decisiones requiere de la existencia de una regla que oriente la evaluación del proceso. Esta regla no es sino un procedimiento lógico y/o matemático que permite comparar las alternativas entre sí, con objeto de su ordenación, de acuerdo a la capacidad mostrada para cumplir cada objetivo. Existen dos procedimientos para el establecimiento de la regla de decisión. El primero de ellos, denominado función de selección, especifica un modelo matemático de contraste, que haga intervenir la participación de los diferentes criterios, con objeto de comparar los resultados alcanzados por las diversas alternativas. Se trataría de transformar los valores correspondientes a cada criterio en valores homogéneos, mediante un método de ponderación, de manera que puedan ser integrados en un resultado común.

Existen diferentes técnicas que permiten la combinación algebraica de los resultados obtenidos por los diversos criterios. Quizás, el más utilizado es método de la suma lineal ponderada. Este procedimiento calcula, para cada alternativa, la suma de los valores correspondientes a los criterios $c_{i j}$, ponderados por los pesos $p_{j}$, de acuerdo a la siguiente ecuación:

$$
r_{i}=\sum_{j=1}^{n} c_{i j} p_{j}
$$

Otras técnicas están basadas en la aproximación a un punto ideal, especificando las coordenadas de un punto ficticio por los valores más altos en todos los criterios. Posteriormente, se calcularian las distancias (euclideas $u$ otras no lineales) entre cada alternativa y dicho punto, con objeto de clasificar los resultados obtenidos, según la proximidad.

El segundo procedimiento se denomina selección heurística y supone la selección exclusiva de algunas alternativas y el rechazo de otras. No obliga a cuantificar cada criterio en cada alternativa, sino que basta con un conocimiento ordinal, que ordene las alternativas (por criterio) de mayor a menor. Existen diversas técnicas capaces de conseguir este objetivo de selección:

- fijar un umbral en alguno de los criterios y eliminar las alternativas que no superen o sobrepasen un determinado valor.

- fijar umbrales en varios criterios y después de ordenar la importancia de éstos, ir eliminando, progresivamente, los que no superen el límite de cada criterio, al analizarlos por orden de importancia.

En un estudio geográfico, la aplicación, sucesiva, de las reglas de decisión permite, en una primera fase, obtener la capa espacial que dé res- 
puesta a un objetivo, por selección multicriterio de los criterios que lo integran, para, posteriormente, realizar la conjunción de objetivos en una capa espacial multiobjetivo (figura 1).

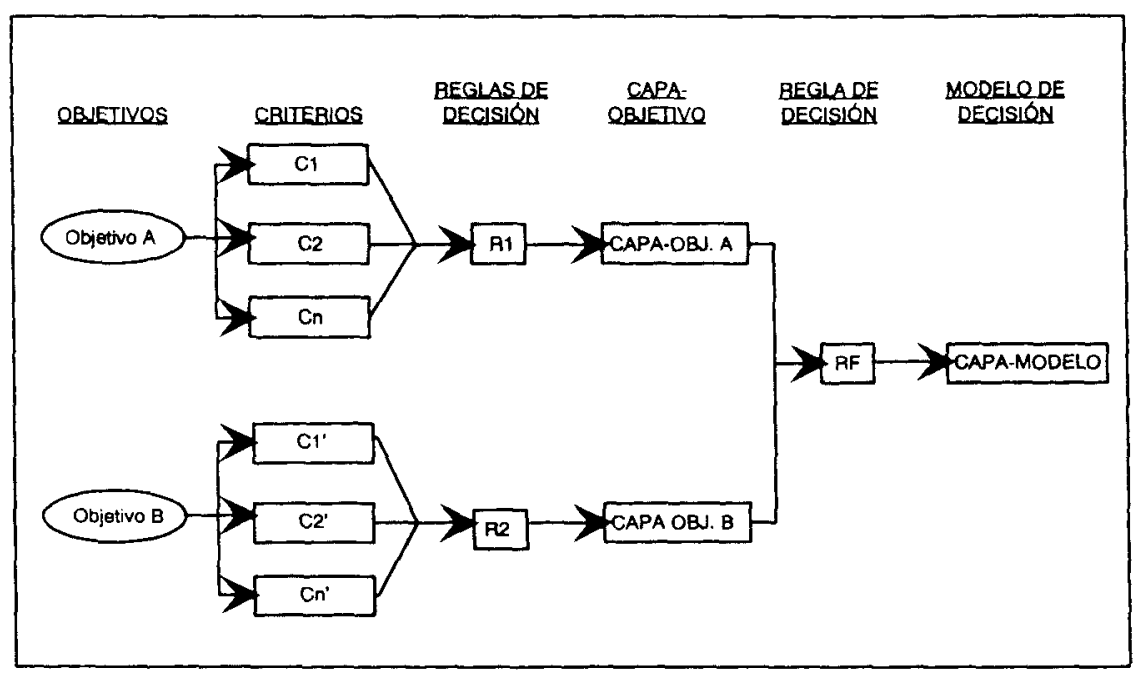

Fuente: BarREdO CANO, 1996, op.cit.

Figura 1. Esquema general del proceso de integración de las diveras fases que componen el procedimiento multicriterio/multiobjetivo.

\section{APLICACIÓN DE LA METODOLOGÍA MULTICRITERIO / MULTIOBJETIVO EN EL PLANTEAMIENTO Y RESOLUCIÓN DE PROBLEMAS MEDIOAMBIENTALES Y TERRITORIALES.}

\subsection{Introducción.}

Una política territorial equilibrada es aquella que pretende la previsión y control de los usos del suelo, mediante una adecuada distribución de las actividades en el espacio. De esta afirmación se desprende la necesidad de conjugar dos realidades contrapuestas: la organización de las actividades humanas en el territorio y la preservación del medioambiente.

Dos son, por tanto, los polos o referencias inevitables de toda política territorial: el medio, como conjunto de factores fisico-naturales, sociales, culturales, económicos o estéticos que es preciso conservar y las actividades humanas, que deben desarrollarse para transformar la naturaleza en beneficio propio. Una actividad humana será ambientalmente aceptable 
en la medida que se adapte y aproveche correctamente la capacidad receptiva del medio y, al mismo tiempo, produzca los mínimos efectos negativos sobre él.

De esta relación mutua entre el medio y la acción del hombre surgen diversos conceptos del máximo interés. Así, el termino aptitud natural se entiende «como la vocación potencial del territorio en función de los recursos naturales que contiene» (Gómez Orea D., 1988). Esta vocación del territorio se manifiesta con referencia a una actividad determinada, de manera que la capacidad de acogida será tanto mejor cuanto que el medio cubra las necesidades locacionales de la actividad en el mayor grado posible.

La armonización de las necesidades humanas con la aptitud del espacio geográfico requiere que el promotor de cualquier proyecto tenga presente la conjunción de las condiciones más favorables del medio y las exigencias o requisitos de la actividad en cuestión, con la finalidad de permitir su desarrollo. Así, la localización de una planta industrial en un área determinada requiere que los elementos de los medios natural (litología, suelo, disponibilidad de agua, etc.) y humano (población, usos del suelo ya existentes, vías de comunicación, etc.) satisfagan las necesidades de esta actividad concreta (capacidad de carga del suelo, baja pendiente topográfica, proximidad a zonas de agua y carreteras, cercanía a otras áreas industriales complementarias, existencia de una mano de obra específica en su alrededor, etc.).

Otro término de gran transcendencia es el de impacto. Se entiende como tal al efecto de una actuación determinada sobre el medio. Mientras en el binomio elementos del medio-actividades, el concepto de aptitud ponía el acento en el primero de los dos componentes, el de impacto lo hace en el segundo.

Este efecto sobre el medio debe ser evaluado no solo por la magnitud sino por su signo. Así, mientras que los impactos positivos realzan la capacidad territorial para acoger las actuaciones humanas (piénsese en el efecto producido por la repoblación forestal sobre la erosión del suelo), los negativos actúan en sentido contrario, reduciendo la potencialidad del medio (piénsese en la destrucción de la vegetación para la instalación de un servicio urbano determinado).

Las causas del impacto pueden ser diversas (Gómez Orea, D., 1992):

- cambios en los usos del suelo.

- emisión de agentes contaminantes. 
- sobreexplotación de recursos naturales y/o ecosistemas.

- subexplotación de recursos naturales y/o ecosistemas.

En el caso del cambio de usos del suelo, debido al desarrollo de la actividad del hombre, el impacto tiene tres orígenes:

a) por la ocupación del suelo natural, destruyendo el soporte de los ecosistemas existentes, como la vegetación y la fauna, modificando diversos aspectos del medio natural como el drenaje interno y superficial, el microclima, el paisaje, etc. Este efecto también se produce por la transformación de algunos usos del suelo (agrarios) a otros de mayor intensidad de ocupación (industriales y urbanos).

b) por efectos inducidos por los cambios de usos del suelo, como la creación de infraestructuras (carreteras, etc.), que pueden originar efectos barrera en el territorio, la contaminación ambiental, etc.

c) por cambios en la distribución de la población, como consecuencia de los nuevos usos implantados, que pueden modificar el equilibrio ambiental en la explotación de los recursos.

\subsection{La correspondencia entre el planteamiento teórico multicriterio/ multiobjetivo y la problemática ambiental y territorial}

De acuerdo a la metodología del planteamiento multicriterio/multiobjetivo, expuesto en apartados anteriores, debemos considerar la posibilidad de establecer una correspondencia entre los elementos implícitos a sus presupuestos teóricos y las derivadas de la problemática medioambiental.

El primer paso consiste en la definición del objetivo u objetivos que guien la investigación. Los motivos de los estudios ambientales pueden ser variados:

- localización de una o varias actividades económicas en el territorio.

- protección de recursos naturales, sociales o culturales.

- recuperación de espacios degradados por la sobreexplotación de recursos.

- minimización de impactos ambientales.

Los objetivos propuestos en un estudio del medio no siempre son complementarios entre sí. Existen, en ocasiones, objetivos conflictivos que exigen la búsqueda de un punto de equilibrio entre metas contradictorias. 
En consecuencia, los estudios promovidos en la consecución de los fines propuestos tratan de racionalizar el empleo del territorio en diversas direcciones:

- Estudios dirigidos a conocer las características del medio con objeto de ordenar los usos del suelo más adecuados a sus peculiaridades. Entran dentro de este grupo los trabajos destinados a la planificación territorial y urbanística y planes de gestión de recursos.

- Estudios dirigidos a conocer las características del medio con objeto de evaluar la posible incidencia ambiental de planes o proyectos. A diferencia de los anteriores, este tipo de trabajos pone el acento en la incidencia ambiental de las actividades en el territorio y no a determinar los usos más adecuados del mismo.

- Estudios dirigidos a conocer las características del medio con objeto de mejorar el aprovechamiento de los recursos.

Una descripción más detallada del tipo de estudios relacionados con los medios natural y humano nos la ofrece W.M.Marsh (Aguilo Alonso, M. et alii, 1992) en el Cuadro I.

\section{Cuadro I. Diferentes tipos de estudios del medio físico}

Inventarios ambientales, temáticos (suelos, pendientes, vegetación,...) o integrados (procesos ambientales, significados,...).

Búsqueda de condiciones favorables y de restricciones ambientales para una actividad.

Zonificación de capacidad, aptitudes, vocaciones, y capacidad de carga, referidas a actividades humanas. Determinación del uso óptimo.

Evaluación de riesgos ambientales. Zonificación de áreas con peligro en caso de usos antrópicos (avenidas, terremotos, movimientos en masa...).

Predicción y evaluación de impacto ambientales. Zonificación de vulnerabilidad y fragilidades territoriales.

Análisis y evaluación de ambientes singulares (zonas húmedas, hábitats únicos,...).

Estudios de localización de actividades, puntuales o extensivas.

Estudios integrados de factibilidad de actividades.

Planificación de infraestructuras y servicios (comunicaciones, sistemas de depuración y abastecimiento, áreas comerciales,...).

Planificación territorial y urbanística y master-plans. Etapas iniciales del proceso de planificación. Formulación de objetivos en relación con los usos del suelo, economía, medio ambiente, demografía, transporte, análisis de condiciones existentes y formulación de escenarios alternativos. Comparación de alternativas en relación con 


\section{Cuadro I. (Continuación)}

objetivos y las condiciones existentes. Elección de la alternativa más adecuada. Recomendaciones y directrices sobre los usos propuestos, sus localizaciones, configuraciones e interrelaciones, y esquema de puesta en práctica, fuentes de financiación, legislación existente o necesaria, cambios en función del tiempo,...

Planes de gestión. Etapas de proyecto en sus fases de diseño, construcción y operación. Estudio y ejecución de medidas correctoras, minimización de efectos ambientales negativos.

Programas de vigilancia y control. Monitoring. Diseño del seguimiento de la eficacia de medidas correctoras, minimización de efectos ambientales negativos.

Los objetivos deben expresarse en los términos más concretos posibles, con la finalidad de ajustar la solución a la problemática planteada en el grado de adecuación óptimo. Así, el objetivo de "promoción de las actividades agrarias" estaría referido de manera excesivamente general y convendría descomponer el mismo en las actividades agrícolas, ganaderas y forestales, e, incluso, en otras subactividades de mayor nivel de detalle: cultivos de secano, ganado bovino, repoblación con frondosas, etc.

El segundo paso supone la definición de los criterios o factores que permitan la evaluación unilateral de cada objetivo. Como hemos podido apreciar en el apartado anterior, la problemática medioambiental y/o territorial presenta objetivos múltiples. A modo de ejemplo, nos vamos a centrar en uno de ellos, del mayor interés en la problemática medioambiental del momento actual: la determinación de la capacidad o vocación def territorio, respecto a la localización de una actividad humana en el espacio.

Los criterios que determinan la localización óptima de una -0 unas-actividad/es concreta/s en el espacio tienen un origen diverso. Gómez Orea (1985) define cuatro tipos de factores capaces de orientar sobre la potencialidad del territorio para acoger una determinada actividad humana:

- Factores relacionados con el medio natural. Entre ellos, podríamos señalar una larga lista de elementos del medio físico, cuya influencia en la localización de las actividades puede ser determinante: la morfología y la pendiente del terreno, la vegetación, el paisaje, el tipo de suelo, etc. Este tipo de variables puede llegar a limitar la instalación de la actividad (piénsese en el establecimiento de la función industrial en terrenos que superen el $10 \%$ de la pendiente o en parajes de alto valor eco- 
lógico, como un parque natural) o incidir positivamente en su establecimiento (áreas llanas, con reducido valor agrológico de los suelos). Alguna de las variables a considerar en este apartado vienen recogidas en el cuadro II.

- Factores basados en el mantenimiento del patrón o modelo locacional existente. Es evidente, que el desarrollo de una determinada actividad resulta mucho más económica si se continúa con el modelo de asentamientos ya establecido. La continuidad espacial o inercia locacional de la industria en un área de estas características, reduce los costos de infraestructura necesarios para su implantación.

- Factores relativos a las características espaciales o esencialmente geográficas, como distancia, accesibilidad, aglomeración, forma y tamaño. La localización óptima de las actividades humanas se encuentra relacionada con la distancia (euclideana) a los principales factores productivos: mercado de mano de obra o de consumo (distancia a la ciudad), materias primas (distancia a la ciudad o a centros productivos), existencia de recursos naturales (como el agua, etc.) o a la propia infraestructura de transporte (distancia a la red de carreteras, ferrocarril, etc.). Si se considera la anisotropia del territorio, cabe referirse a la accesibilidad, como a la mejor manera de medir distancias mínimas, entre lugares, considerando los costos de desplazamiento sobre el territorio de forma proporcional a la impedancia del terreno al movimiento de personas, mercancias, etc. Finalmente, la forma y el tamaño de las parcelas de terreno, que cumplan las condiciones impuestas por el resto de los factores de localización, resultan fundamentales para concluir si es o no factible la instalación de determinadas actividades humanas, que exigen un espacio mínimo para su organización y desarrollo.

Cuadro II. Algunas variables del medio físico y su relación con el establecimiento espacial de las actividades humanas

\begin{tabular}{ll}
\hline VARIABLE & $\begin{array}{l}\text { CONDICIONANTES PARA EL ESTABLECIMIENTO DE } \\
\text { ACTIVIDADES HUMANAS EN EL ESPACIO }\end{array}$ \\
\hline geología & \\
tipo de roca madre & - riesgo sísmico \\
(características físico- & capacidad portante o de carga \\
químicas). & existencia de acuíferos \\
& - estabilidad de taludes \\
& existencia de materiales de construcción \\
& drenaje del terreno \\
\hline
\end{tabular}


Cuadro II. Algunas variables del medio físico y su relación con el establecimiento espacial de las actividades humanas (continuación)

\begin{tabular}{|c|c|}
\hline VARIABLE & $\begin{array}{l}\text { CONDICIONANTES PARA EL ESTABLECIMIENTO DE } \\
\text { ACTIVIDADES HUMANAS EN EL ESPACIO }\end{array}$ \\
\hline $\begin{array}{l}\text { geomorfología } \\
\text { - formas topográficas } \\
\text { - altitud } \\
\text { - exposición solar } \\
\text { - pendiente }\end{array}$ & $\begin{array}{l}\text { - zonas inundables } \\
\text { - topografía llana o abrupta } \\
\text { - insolación }\end{array}$ \\
\hline $\begin{array}{l}\text { edafología } \\
\text { - tipos de suelos } \\
\text { - textura } \\
\text { - profundidad, etc. }\end{array}$ & $\begin{array}{l}\text { - capacidad agrológica } \\
\text { - tipos de especies a cultivar }\end{array}$ \\
\hline $\begin{array}{l}\text { condiciones } \\
\text { climáticas } \\
\text { - variables climáticas } \\
\left(\dagger^{\circ} \text {, precipitación, }\right. \\
\text { H, etc.) }\end{array}$ & $\begin{array}{l}\text { - existencia de agua } \\
\text { - potencialidad de inundaciones. } \\
\text { - condiciones ambientales atmosféricas (bienestar climático) }\end{array}$ \\
\hline vegetación & $\begin{array}{l}\text { - estabilidad de pendientes } \\
\text { - microclimas locales } \\
\text { - calidad del paisaje } \\
\text { - hábitat animal }\end{array}$ \\
\hline medio acuoso & $\begin{array}{l}\text { - recursos recreativos } \\
\text { - calidad del paisaje }\end{array}$ \\
\hline
\end{tabular}

- Factores basados en otro tipo de condicionantes locacionales. Podríamos incluir en este grupo a factores tan diversos como la tradición, el nivel de desarrollo económico, las decisiones políticas de carácter territorial o las preferencias personales. Aunque, en muchas ocasiones, este tipo de variables resulten difíciles de medir, por su indudable peso de subjetividad, no deja de ser considerada su importancia en todo proceso de decisión humana.

Finalmente, la evaluación del conjunto del proceso y la conjunción de objetivos y criterios exige de la existencia de una regla que permita valorar, previamente, la contribución individual de cada uno de los factores, para integrar los resultados después de su homogeneización. Esta operación puede realizarse mediante una función de selección, que establezca de forma lógica y/o matemática la medición de las aportaciones parciales de los factores y su adición ponderada. 
La evaluación espacial del territorio exige, así mismo, la definición previa de las alternativas o áreas elementales disponibles donde comparar la potencial localización de la actividad considerada. Esta definición de las unidades geográficas, que sirvan como soporte de recogida y contraste de la información, resulta esencial en la valoración metodológica global. Existen diferentes modelos de compartimentación del espacio, que permiten la definición de las unidades espaciales objeto del análisis. El modelo raster, que divide el espacio en celdillas regulares, en general cuadradas, se ha mostrado como un eficaz sistema de referencia espacial al permitir almacenar la información geográfica en formato digital regular, lo que facilita su posterior tratamiento, como tendremos ocasión de comprobar.

La aplicación de la metodología implícita al modelo multiobjetivo/multicriterio supone la comparación, para cada objetivo, de los valores obtenidos en las diversas alternativas (unidades espaciales) por los criterios del análisis. Las fases de este procedimiento serían las siguientes:

- Superponer una retícula cuadrada sobre el área de estudio, con objeto de recoger la información de las variables ambientales que van a servir para la elaboración del mapa de aptitud territorial. El tamaño o resolución de la malla incide, directamente, en la precisión del análisis. Si la malla es de tamaño reducido, necesitaremos mayor número de celdas para cubrir la zona a estudiar, con lo que será más laboriosa la investigación. Lo contrario ocurriría con una retícula de mayor tamaño.

- Definir una serie de criterios o factores básicos para la valoración de la aptitud del territorio a examinar. Un ejemplo nos puede servir para facilitar la explicación. Si deseáramos evaluar la aptitud de un área concreta para la repoblación forestal, deberíamos seleccionar aquellas variables geográficas que se supongan decisivas en la potenciación de esta actividad económica determinada: vegetación, pendiente, exposición, litología, altitud, etc.

- Ponderar los diferentes criterios seleccionados, según una escala de medida, en consonancia con la aptitud a cuantificar. La medición debe establecerse siempre en idéntica dirección para todos los criterios (por ejemplo, que los valores reducidos supongan una contribución a que la aptitud sea baja y los valores elevados a que sea alta). Se aconseja que los rangos de todas las variables sean comunes. Así, si una variable se mueve entre valores de 1 a 10, el resto de las variables debería seguir la misma norma.

- Digitalizar los valores de los diversos criterios, después de superponer la malla cuadrada sobre el territorio a analizar. El resultado será la obtención de una serie de capas, en formato raster, para las diferentes variables, donde se represente, en cada celda el valor correspondiente a la digitalización o relación existente entre cada criterio y el territorio. 
- Definir, mediante criterios limitantes, las áreas del territorio donde resulta prácticamente imposible establecer la actividad programada. Piénsese en la exclusión de la actividad industrial sobre el espacio ocupado por un lago o por un parque natural. Estos criterios o factores, que excluyen la posibilidad de acometer una actuación, de manera absoluta, se denominan limitantes.

- Integrar las diferentes capas de información, relativas a los diversos criterios, de acuerdo a una regla previamente establecida, de manera que pueda referirse a un modelo de decisión perfectamente definido (figura 2). En nuestro caso, hemos seleccionado la suma ponderada de los factores o criterios. La figura 3 muestra, a modo de ejemplo, la integración de dos

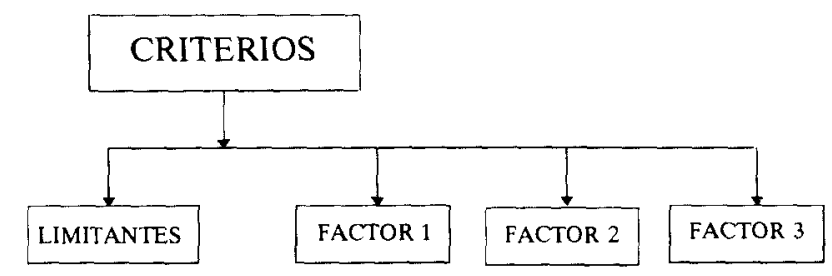

Fuente: E.P.

Figura 2. Esquema de integración de los factores y criterios limitantes en un planteamiento de un único objetivo.

CAPA A

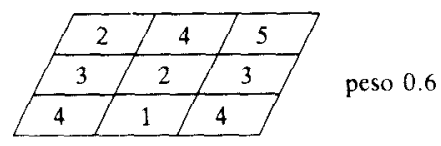

CAPA B

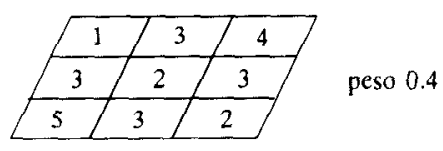

CAPA

TOTAL

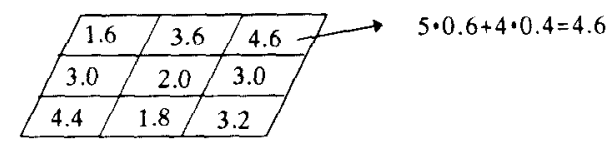

Fuente: E.P.

Figura 3. Integración de dos capas de información, relativas a los criterios $A$ y $B$, ponderadas de forma desigual (pesos 0.6 y 0.4 ). 
capas con retícula espacial de $3 \times 3$ celdas, ponderando los criterios con pesos respectivos de 0.6 y 0.4 .

- Clasificar los valores de la integración anterior en un reducido número de categorías, que expresen el valor de la aptitud de forma más sintética.

\section{LOS SISTEMAS DE INFORMACIÓN GEOGRÁFICA RASTER Y SU CAPACIDAD DE HACER OPERATIVO, INFORMATICAMENTE, EL MODELO TEÓRICO DE LA EVALUACIÓN MULTICRITERIO}

Los sistemas de información geográfica no son sino programas o conjuntos de programas informáticos diseñados para trabajar con información georreferenciada, mediante coordenadas espaciales o geográficas. El estudio simultáneo de los aspectos temático y espacial de la información geográfica permite un análisis más complejo de las estructuras espaciales.

El manejo de los SIG plantea un nuevo concepto de la estructura espacial, ligado a la modelización del espacio, mediante una representación digital en base a objetivos discretos. De los dos modelos o sistemas existentes, vectorial y raster, el segundo compartimenta el espacio en una retícula regular, cuyos objetos poligonales sirven de elementos espaciales de recogida de la información. Pues bien, a partir de estos elementos espaciales simples se puede gestionar informaticamente la información geográfica con un elevado indice de eficacia. Es este uno de los motivos fundamentales del interés de su empleo para gestionar la problemática medioambiental del territorio en el marco del planteamiento teórico multicriterio.

La integración de los SIG raster y la metodología multicriterio genera una potente herramienta de análisis, que a pesar de las limitaciones sugeridas por diversos autores, que tienen que ver con el elevado número de alternativas a evaluar en un formato espacial de esta naturaleza (piénsese que una capa espacial de $500 \times 500$ supone un universo a analizar de 250.000 celdillas), ofrece la posibilidad de intervenir en los complejos procesos del análisis espacial: asignación y localización de actividades teniendo presente diversos criterios y múltiples objetivos.

Los sistemas de información geográfica raster permiten piantear operaciones que reproducen los pasos de la metodología multicriterio, lo que facilita la selección de las alternativas espaciales acordes con la localización óptima de una actividad humana, o cualquier otro problema medioambiental, en un tiempo record.

Veamos más detenidamente como operan los SIG raster en el contex- 
to procedimental definido en el apartado anterior:

- el formato raster, propio de este modelo de sistemas, garantiza el tratamiento de la información geográfica a partir de la retícula espacial cuadrada. Cada variable, representativa de un criterio o factor del análisis, queda recogida en una capa de información individualizada. La fuente de información, sin duda, más interesante procede de las imágenes de satélite o teledetección. Periódicamente, mediante sensores remotos, se registra en formato digital la radiación emitida por la superficie de la Tierra. Su posterior tratamiento permite disponer de un número apreciable de variables geográficas (usos del suelo, calidad del agua, estado de las cosechas, etc.).

- la información de cada capa o variable puede ser reclasificada y normalizada de manera que los criterios del análisis queden preparados para su integración posterior, según una escala uniforme de categorías, previamente, establecida. Los criterios pueden ser de dos tipos: factores (que reflejan en cada celda o pixel del territorio un valor digital correspondiente al fenómeno evaluado, en una escala homogénea 0-10, 0-100. etc.) y los criterios limitantes (imágenes booleanas o binarias, de valores 0 y 1 , que reflejan mediante ceros la superficie del territorio limitada al establecimiento de la actividad considerada).

- la integración de las diferentes capas se realiza mediante operaciones de cálculo algebraico y superposición lógica y/o matemática de los diferentes estratos de información (multiplicación por un escalar, suma, resta, etc.).

- los valores de la capa resultante pueden ser reclasificados, con objeto de obtener un mapa temático final de la evaluación de la aptitud territorial en el establecimiento de la actividad considerada.

\section{ANÁLISIS DE UN CASO PRÁCTICO DE LA APLICACIÓN DE LA METODOLOGIA MULTICRITERIO, MEDIANTE SIG RASTER, RESPECTO AL ESTABLECIMIENTO DE UNA ACTIVIDAD HUMANA EN EL TERRITORIO}

\subsection{Planteamiento del problema}

Se trata de exponer, como la metodología EMC puede aplicarse en la resolución de una problemática medioambiental y/o territorial determinada. La localización de la industria se halla afectada por la consideración de un variado conjunto de causas, relacionadas, tanto con la proximidad a los factores productivos (distancia mínima a los centros de producción o de 
elaboración de las materias primas y al mercado de consumo), como por la cercania a las principales vias de transporte (carretera y ferrocarril, principalmente) o a las características del medio físico (topografía llana, etc.:). El problema que nos planteamos resolver es el de la localización óptima de la actividad industrial en un territorio concreto (objetivo único) de acuerdo a un conjunto de criterios y factores limitantes (multicriterio). Los criterios a considerar han sido los que se detallan a continuación:

\section{criterios}

- Distancia mínima a la ciudad (mercado de consumo y mano de obra).

- Distancia mínima a la red de carreteras.

- Distancia mínima a los lagos o embalses (al agua como recurso natural necesario para el proceso industrial).

- Superficie de terreno superior a 3 Has.

\section{factores limitantes}

- Exclusión del área urbanizada.

Relieve
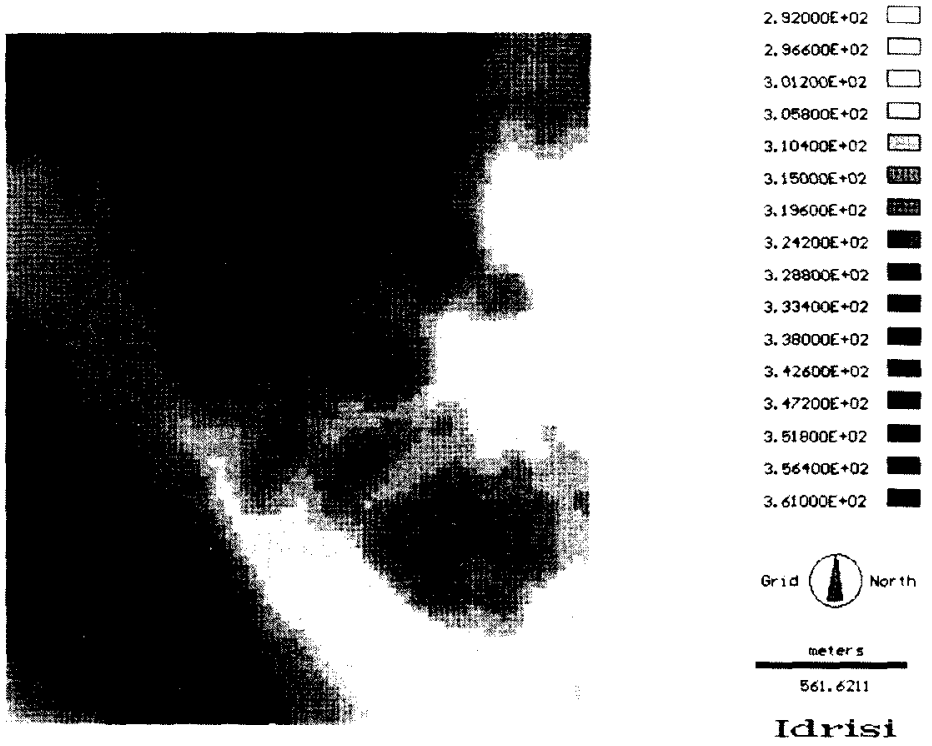

Fuente: E.P.

Figura 4. Topografía del terreno de la zona de estudio. 
- Topografía llana (pendiente inferior al 10\%).

Se dispone, para desarrollar el ejemplo seleccionado, de dos capas de información geográfica, relativas a un modelo digital del terreno (mapa de altitudes), que denominaremos RELIEVE y a los usos del suelo, que denominaremos USOSUELO ${ }^{2}$. Ambos estratos de información se hallan organizados según un modelo raster de 86 filas y 72 columnas, con una resolución de 30 metros. Las figuras 4 y 5 muestran la topografía del terreno

Usos del suelo
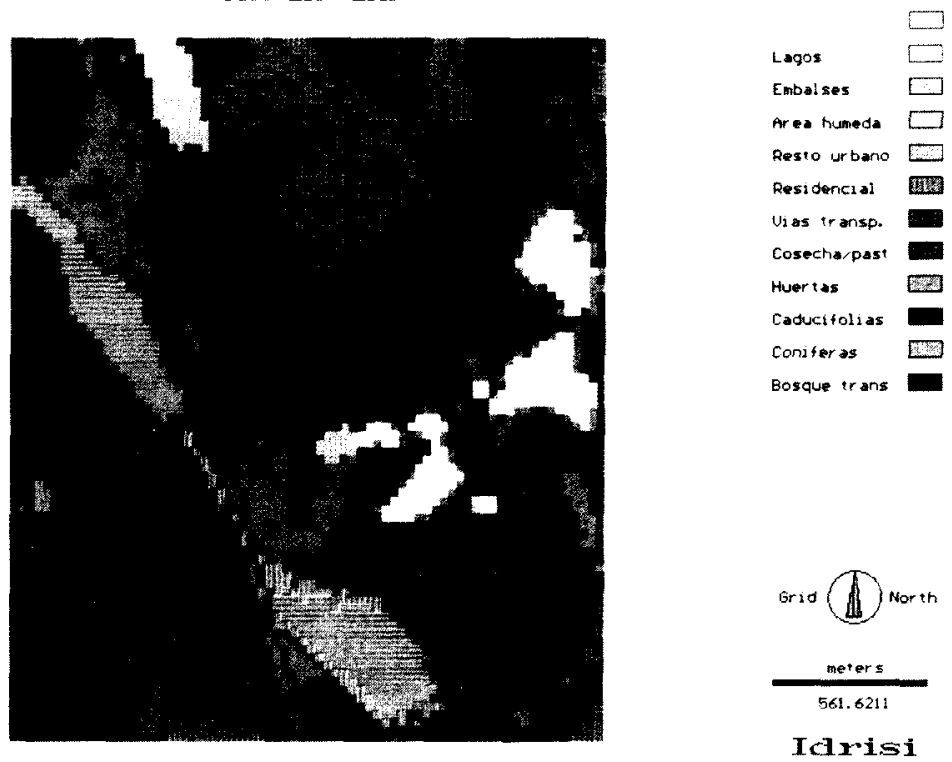

Fuente: E.P referimos.

y la distribución espacial de usos del suelo a que nos

Figura 5. Usos del suelo de la zona de estudio.

Para la consecución del objetivo previsto, hemos utilizado el S.I.G. raster IDRISI, según la versión 4.1. Las diversas operaciones inherentes a este sistema informático permiten realizar las fases del trabajo de acuerdo a la metodología EMC, tal como ha sido presentada en anterio-

2 Los dos estratos de información utilizados se han obtenido de los ficheros incluidos en el programa IDRIDI, versión 4.1. 
res apartados.

\subsection{Resolución del problema planteado}

La aplicación del método multicriterio/multiobjetivo a la localización óptima de la función industrial, mediante la formalización lógico matemática de un SIG raster, exige la cumplimentación sucesiva de los siguientes pasos:

- Cuantificar los criterios de valoración establecidos (distancia mínima a la red de carreteras, ciudad y zonas de agua), mediante capas de información individualizadas para cada uno de ellos.

- Delimitar las áreas limitadas a la ubicación de la industria (espacio urbanizado y situado sobre las zonas de agua y de pendiente superior al $10 \%$ ), mediante capas de información individualizadas para cada factor limitante o excluyente.

- Superponer algebraicamente las capas de información, relativas a los criterios, afectadas por un peso proporcional a su participación (en nuestro caso, hemos definido pesos iguales).

- Excluir del territorio de análisis el espacio que no cumpla las condiciones limitantes.

- Ordenar las alternativas del territorio resultante, de acuerdo a la mayor o menor capacidad de satisfacción de los criterios definidos en el análisis (del pixel o celdilla de valor máximo al de valor mínimo).

- Delimitar la zona de máxima satisfacción de los criterios de 3 Has. de superficie.

Las diversas fases establecidas pueden ser ejecutadas, aplicando las operaciones elementales de que dispone IDRISI. Siguiendo idéntico proceso al elaborado anteriormente la concreción del mismo sería la siguiente:

\section{a) Cuantificación de los criterios de valoración}

Los factores o criterios básicos de valoración de la óptima localización de la industria son la distancia mínima a la ciudad y la red de carreteras y a las zonas de agua. De manera separada, la cuantificación de los mismos sería:

Distancia mínima a la ciudad y la red de carreteras.

Los operaciones a ejecutar mediante IDRISI serían las siguientes:

- Reclasificar la imagen de usos del suelo en una imagen binaria, des- 
tacando los usos 5 y 6 , suelo urbano y de transporte, con el valor 1 y el resto del territorio con 0 (comando Reclass).

- Hallar la distancia desde el área definida anteriormente (ciudad y carreteras) al resto del territorio (comando Distance).

- Normalizar los valores anteriores entre 0 y 100 (comando Stretch).

- Invertir la imagen anterior, de manera que las áreas más próximas tengan valores superiores y las más alejadas más bajos (comandos Initial y Overlay).

Valoracion de distancia (ciudad $\mathbf{y}$ carreteras)
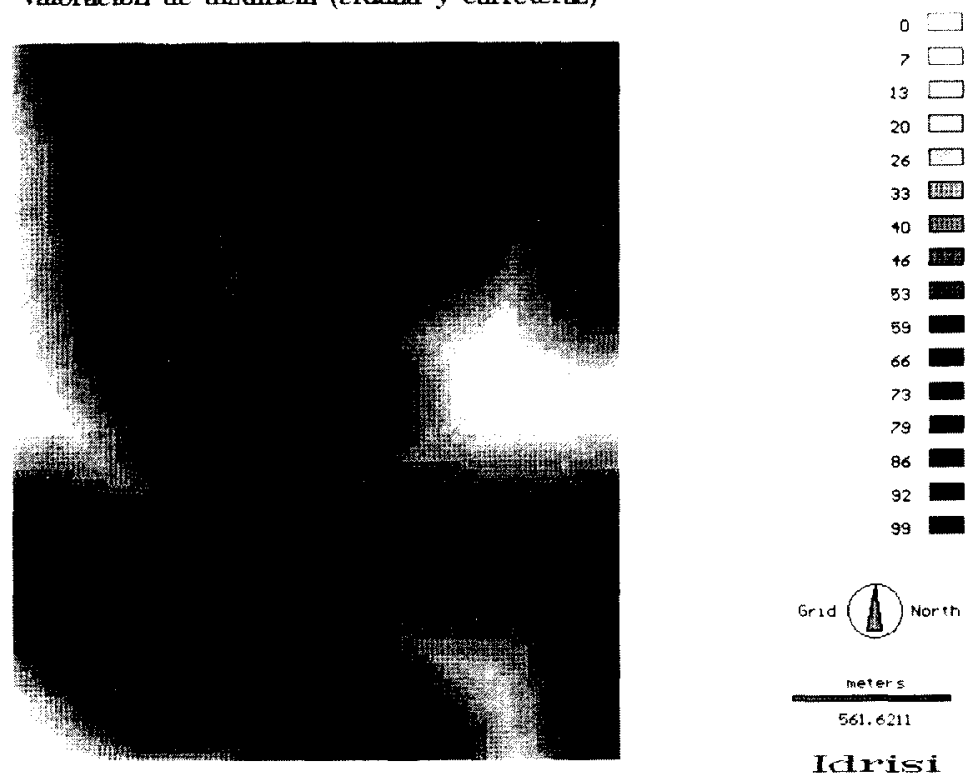

Fuente: E.P.

La imagen resultante de la evaluación de este primer factor sería la de la figura 6.

Figura 6. Valoración cuantitativa del primer criterio (distancia a la ciudad y red de carreteras).

Distancia mínima a las zonas de agua

Siguiendo un proceso semejante para la valoración de la distancia a los 
Valoracion de distancia (embalses y lagos)
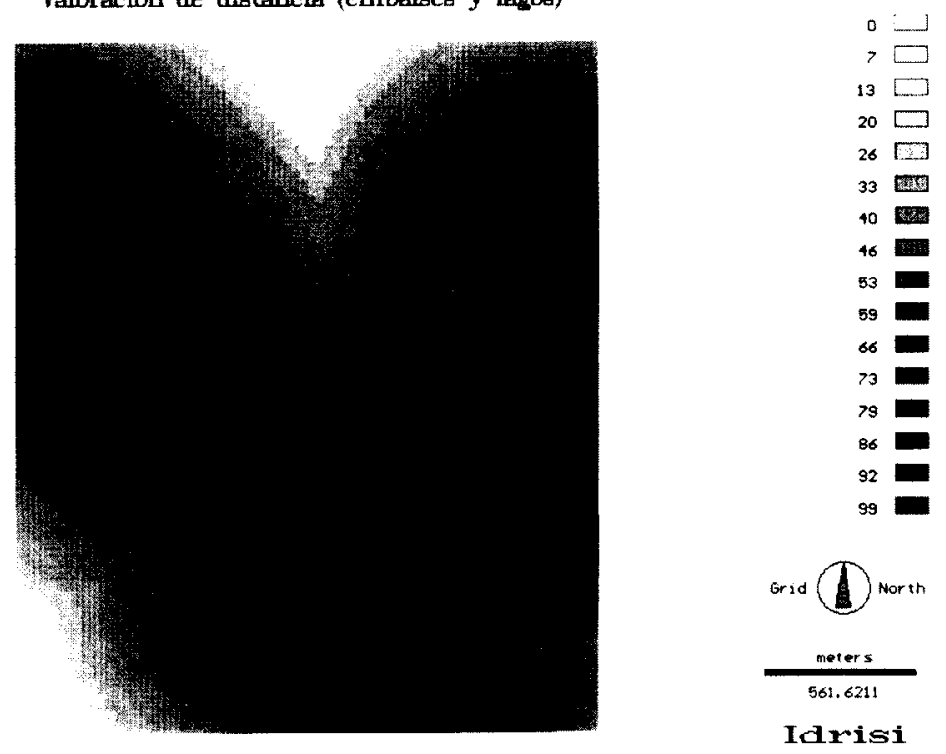

Fuente: E.P.

Figura 7. Valoración cuantitativa del segundo criterio (distancia a los lagos y embalses).

embalses y lagos existentes en el territorio de análisis obtendríamos la imagen de la figura 7.

b) Delimitar las áreas excluidas de la localización de la industria

La demarcación de las zonas limitadas al establecimiento de la función industrial se realizan de acuerdo al factor limitante que las justifica. En el caso que nos ocupa, las áreas excluidas por pertenecer al suelo urbanizado o de zona de agua se obtienen, reclasificando la imagen de los usos del suelo, destacando con el valor 0 el área a considerar y con el valor 1 el resto del territorio (comando Reclass). En el caso de las pendientes, es preciso calcular, previamente, la imagen de los desniveles relativos, a partir del modelo digital del terreno (comando Surface), reclasificando con el valor 0 , el territorio cuya pendiente sea superior al $10 \%$ y el resto con el valor 1 .

c) Superposición algebraica de las capas relativas a los criterios y a los 
factores limitantes

En el Sistema de Información Geográfico IDRISI, la superposición ponderada de los criterios y la de los factores limitantes se realiza a partir de un comando, denominado MCE, que ha sido diseñado para la aplicación de la técnica de evaluación multicriterio. Se trata de sumar algebraicamente los

Valoracion final
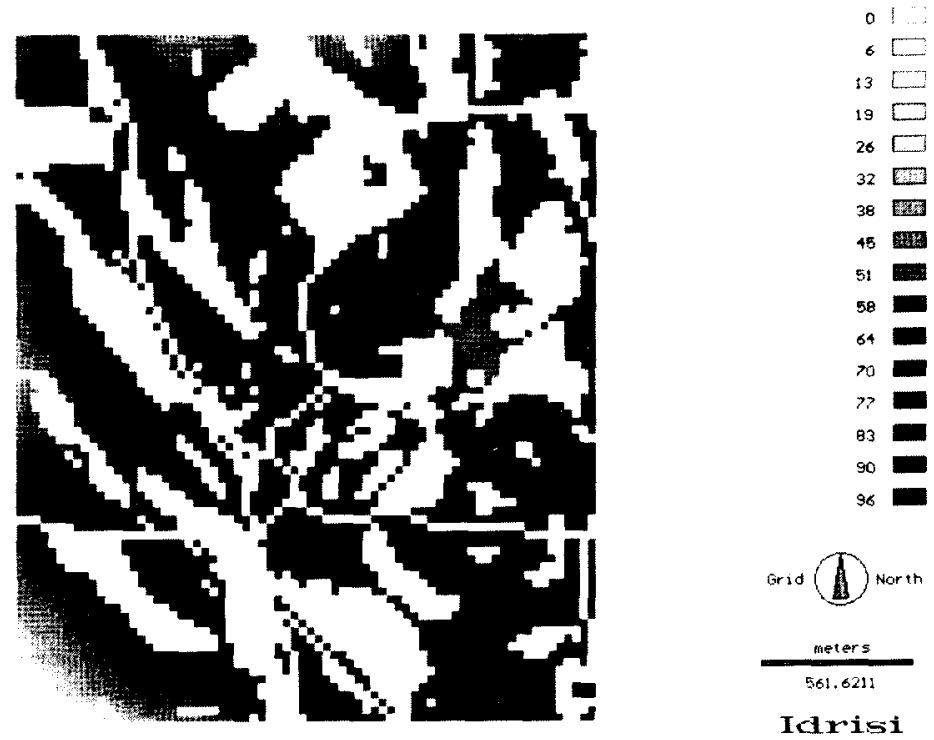

Fuente: E.P.

valores de las capas de información, relativas a los cri-

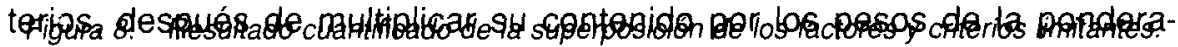
ción, superponiendo, de manera excluyente, las áreas urbanizadas, de agua o de pendiente superior al $10 \%$. El resultado final queda reflejado por la figura 8.

d) Ordenación de las alternativas del territorio, según la cuantificación efectuada, y delimitación del terreno de localización óptima

El comando Rank de IDRISI, permite la ordenación de los pixeles o celdas de una capa de información desde el valor más elevado al más reducido, identificando con el valor 1 al más alto, con el 2 al siguiente y así sucesivamente (se puede introducir un segundo criterio de ordena- 
ción en el caso de que los valores a ordenar coincidan). Esta operación facilita la delimitación del área óptima, reclasificando el fichero de información después de su ordenación. En el caso que nos ocupa, se trataría de reclasificar con el valor 1 , una determinada porción del espacio (por ejemplo 30 Has., que equivaldrian a los primeros 333 pixeles, ya que la superficie de cada uno equivale a $30 \times 30$ metros, o a 0.09 Has.) y el resto con el valor 0 .

El resultado final del área buscada se hallaría, identificando las zonas
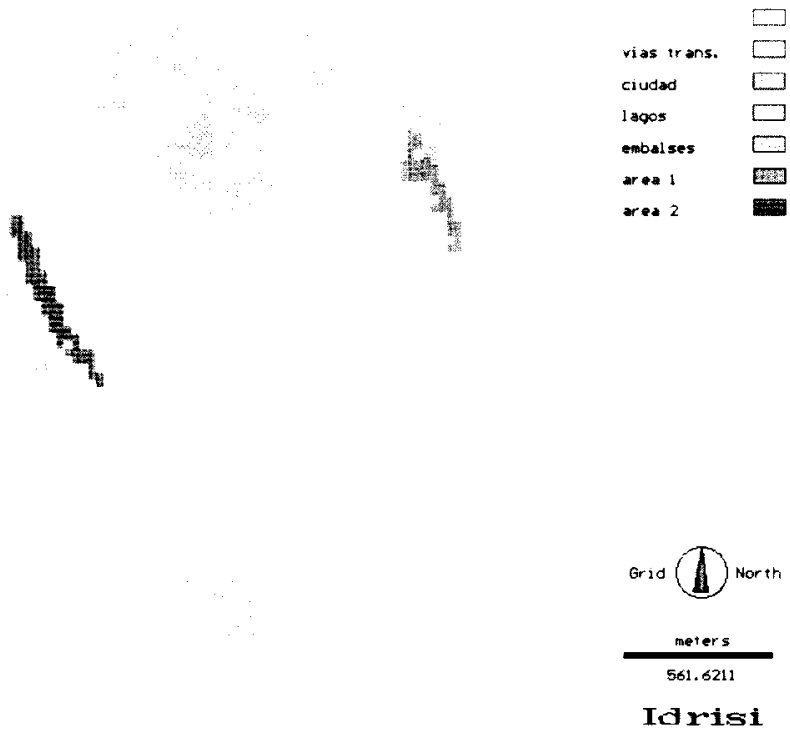

Fuente: E.P.

existentes (espacios homogéneos y contiguos) (comandos Group y Area) que en nuestro caso daría lugar a dos terrenos de 3.33 y $5.04 \mathrm{Has}$, respectivamente. La imagen de las prigurales refleja la posicion de las áreas de localización óptima, respecto a los principales factores de localización analizados.

\section{CONCLUSIONES}

El paradigma multicriterio/multiobjetivo ofrece un marco teórico de gran interés en los diversos campos de estudio, donde la toma de decisiones es 
fundamental. Su capacidad analítica, intrinseca a un planteamiento de esta naturaleza, convierte a las técnicas EMC en una poderosa herramienta de asistencia a los procesos de decisión, basada en la multidimensionalidad de criterios y puntos de vista que afecta la compleja problemática del mundo actual.

Su aplicación a la problemática territorial y/o medioambiental ha sido efectuada con gran éxito, merced a la eficacia de los Sistemas de Información Geográfica. La individualización de las alternativas espaciales y su evaluación respecto a alcanzar determinadas metas en el campo de la ordenación territorial y problemática medioambiental ha sido utilizada con vistas a lograr una equilibrada distribución de las actividades en el territorio.

La adecuada integración del planteamiento multicriterio y de los S.I.G. raster, mediante un ejemplo concreto resuelto con Idrisi, ofrece una perspectiva de como ambas herramientas pueden ser empleadas en la evaluación de soluciones específicas a la problemática territorial y/o medioambiental.

\section{BIBLIOGRAFIA}

Aguilo Alonso, M. ET ALII (1992): Guía para la elaboración de estudios del medio físico. CEOTMA. Ministerio de Obras Públicas y Transportes. Madrid, 808 págs.

BarRedo Gano, J.I. Y Bosque Sendra, J. (1995): "Integración de evaluación multicriterio y Sistemas de Información Geográfica para la evaluación de la capacidad de acogida del territorio y la asignación de usos del suelo". Actas del IV Congreso español de Sistemas de Información Geográfica. Madrid, págs. 191-200.

Baraedo Cano, J.I. (1996): Sistemas de Información Geográfica y Evaluación Multicriterio. Edit. Rama. Madrid, 264 pág.

Bosque Sendra, J. (1992): Sistemas de Información Geográfica. Edit. Rialp. Madrid, 451 págs.

Bosque Sendra, J., Escobar Martinez, F.J., Garcia Hernandez, E. y Salado Garcia, M.J. (1994): Sistemas de Información Geográfica: Prácticas con PC ARC/INFO e IDRISI. Edit. Rama. Madrid, 478 págs.

Coinesa FERnÁndez-VItoria, V. (1997): Guía Metodológica para la Evaluación del Impacto Ambiental. Edit. Mundi-Prensa. Madrid, 412 págs.

EASTMAN, J.R. (1993): IDRISI, A grid based geographic analisys system. Version 4.1. Clark University, Worcester, Massachusetts.

EAstman, J.R., KYeM, P.A., TOledano, J. y JIN, W. (1993): Gis and Decision Making. UNITAR. Ginebra, 112 págs.

Estevan Bolea, M.T. (1984): Evaluación del Impacto Ambiental. Madrid.

Gómez OREA; D. (1985): El espacio rural en la ordenación del territorio. Instituto de Estudios Agrarios, Pesqueros y Alimentarios. Madrid.

Gomez OreA, D. (1988): Evaluación del impacto ambiental de proyectos agrarios. Ministerio de Agricultura Pesca y Alimentación. Madrid, 286 págs.

Gomez OreA, D. (1992): Evaluación del impacto ambiental. Edit. Agricola Española. Madrid.

Gutiérrez Puebla, J. y Gould, M. (1994): Sistemas de Información Geográfica. Edit. Sintesis. Madrid, 251 págs.

HeRnANDEZ FERnANDEZ, S. (1995): Ecología para Ingenieros. El Impacto Ambiental. Colegio de Ingenieros de Caminos, Canales y Puertos. Madrid, 427 págs.

JANKOWSKI, J. (1995): "Integrating geographical information system and multi-criteria decisionmaking methods». Int. J. Geographical Information Systerns, vol. 9, nº 3, págs. 251-273.

MALCZEWSKI, J. (1996): A GIS based approach to multiple criteria group decision-making". int. J. Geographical Information Systems, vol. 10, nº 8, págs. 955-971. 